\title{
The stellar environment of SMC N81 ${ }^{\star}, \star \star$
}

\author{
M. Heydari-Malayeri ${ }^{1}$, F. Meynadier ${ }^{1}$, V. Charmandaris ${ }^{2,1}$, L. Deharveng ${ }^{3}$, Th. Le Bertre ${ }^{1}$, \\ M. R. Rosa ${ }^{4, \star \star \star}$, and D. Schaerer ${ }^{5,6}$
}

1 LERMA, Observatoire de Paris, 61 avenue de l'Observatoire, 75014 Paris, France

2 Cornell University, Astronomy Department, 106 Space Sciences Bldg., Ithaca, NY 14853, USA

3 Observatoire de Marseille, 2 place Le Verrier, 13248 Marseille Cedex 4, France

${ }^{4}$ Space Telescope European Coordinating Facility, European Southern Observatory, Karl-Schwarzschild-Strasse-2, 85748 Garching bei München, Germany

5 Observatoire de Genève, 51 Ch. des Maillettes, 1290 Sauverny, Switzerland

${ }^{6}$ Laboratoire d'Astrophysique, UMR 5572, Observatoire Midi-Pyrénées, 14 avenue E. Belin, 31400 Toulouse, France

Received 15 May 2003 / Accepted 25 August 2003

\begin{abstract}
We present near infrared JHK imaging of the Small Magellanic Cloud compact H II region N81 using the ISAAC camera at the ESO Very Large Telescope (Antu). Our analysis of the stellar environment of this young massive star region reveals the presence of three new stellar populations in the surrounding field which are mainly composed of low mass stars. The main population is best fitted by evolutionary models for $\sim 2 M_{\odot}$ stars with an age of 1 Gyr. We argue that these populations are not physically associated with the H II region N81. Instead they are the result of a number of low mass star forming events through the depth of the SMC south of its Shapley's wing. The populations can rather easily be probed due to the low interstellar extinction in that direction.
\end{abstract}

Key words. stars: early-type - ISM: individual objects: N81 (SMC) - galaxies: Magellanic Clouds

\section{Introduction}

High-Excitation Blobs (HEBs) represent a rare class of compact $\mathrm{H}$ II regions in the Magellanic Clouds (Heydari-Malayeri \& Testor 1982). In contrast to the typical H II regions of these neighboring galaxies, which are extended structures spanning several minutes of arc on the sky (more than $50 \mathrm{pc}$ ) and powered by a large number of hot stars, HEBs are dense small regions usually $5^{\prime \prime}$ to $10^{\prime \prime}$ in diameter ( 1 to $3 \mathrm{pc}$ ). Moreover, they happen to lie adjacent or in the direction of the typical giant H II regions, with the exception of SMC N81 (Henize 1956) which has apparently been formed in isolation. They are probably the optical counterparts of the Galactic ultracompact H II regions (Churchwell 1990) lying relatively close to the cloud surface because the molecular cloud layers above the newborn stars have been scraped by the strong UV field

Send offprint requests to: F. Meynadier,

e-mail: Frederic. Meynadier@obspm. fr

* Based on observations obtained at the European Southern Observatory, Paranal, Chile; Program 69.A-0123(A).

$\star \star$ Based on observations made with the NASA/ESA Hubble Space Telescope, obtained at the Space Telescope Science Institute, which is operated by the Association of Universities for Research in Astronomy, Inc., under NASA contract NAS 5-26555. These observations are associated with program \# 6535 .

$\star \star \star$ Affiliated to the Astrophysics Division, Space Science Department of the European Space Agency. of the adjacent massive stars or by the champagne flows (Tenorio-Tagle et al. 1979).

Optical observations of a number of these objects, LMC N159-5, N160A1, N160A2, N83B, N11A, as well as SMC N88A and N81, allowed one to derive their global physical characteristics and establish them as a particular class of metal-poor H II regions in the Magellanic Clouds (Heydari-Malayeri \& Testor 1982; Heydari-Malayeri \& Testor 1983, 1985, 1986; Heydari-Malayeri et al. 1990; Testor \& Pakull 1985; Heydari-Malayeri et al. 1988). In particular, it was shown that HEBs are generally very affected by local dust (references above; see also Israel \& Koornneef 1991). However, those early studies made it also clear that in order to better understand the stellar properties in those small regions, sub-arcsecond spatial resolution was needed.

This was achieved by our HST WFPC2 high resolution imaging of seven HEBs, SMC N81, N88A, LMC N159-5, N83B, N11A, N160A1, N160A2 (Heydari-Malayeri et al. 1999a,b,c, 2001a,b, 2002a,b). We were able to spatially resolve these objects for the first time, uncovering their morphology, nebular features, the location of their high excitation [O III] $\lambda 5007$ zones, and the variation of the extinction across them. Those observations showed that powerful stellar winds and shocks create magnificent scenes testifying to high activity: outstanding emission ridges, cavities sculpted in the ionized gas, prominent dust structures protruding from hot gas, 
and even unknown compact $\mathrm{H}$ II blobs immersed in the HEBs and harboring very young hot stars. In a few cases our HST images revealed some of the exciting stars, but not all of them, and in some other cases we could not conclusively identify the exciting stars at all. This is likely due to the high dust content of those regions, which is rather remarkable given their low metallicity (Bouchet et al. 1985).

The HII region N81 lies well outside the main body of the SMC in the outer parts of Shapley's wing. Discovered by Shapley (1940) as a "large cloud of faint stars extending eastward from the SMC to the LMC", the wing was shown to be in fact the tail of a much larger H I structure linking the SMC to the LMC (Kerr et al. 1954; Hindman et al. 1963; Mathewson $\&$ Ford 1984). Models and observations suggest that the neutral hydrogen structures known as the Magellanic Bridge, the Magellanic Stream, and the Leading Arm result from the Clouds' interaction with each other and the Milky Way (Murai \& Fujimoto 1980; Moore \& Davis 1994; Gardiner \& Noguchi 1996; Putman et al. 1998). Several works support the finding that the SMC wing is pointing towards the LMC, and is therefore closer to us than the SMC bar (Mathewson et al. 1986; Caldwell \& Coulson 1986; Irwin et al. 1990). Regarding N81 itself, which lies towards the outer parts and south of the wing, our HST observations showed the presence of a tight cluster of newborn massive stars embedded in this nebular "blob" of $\sim 10$ " across (Heydari-Malayeri et al. 1999c, hereafter Paper I). Six of them are grouped in the core region of $\sim 2$ " diameter, with two of the main exciting stars, in the very center, separated by only 0.27 or $0.08 \mathrm{pc}$. The images display violent phenomena such as stellar winds, shocks, ionization fronts, typical of turbulent starburst regions. Follow-up far UV spectroscopy with STIS (Heydari-Malayeri et al. 2002b, Paper II) also revealed a particularly interesting case in SMC N81, where several of the stars are O6-O8 types, but display extremely weak wind profiles (Martins et al. 2002). The astonishing weakness of their wind profiles and the sub-luminosity (up to $\sim 2$ mag fainter in $M_{V}$ than the corresponding dwarfs) make these stars a unique stellar population in the Magellanic Clouds. Our analysis suggests that they are probably in the Hertzsprung-Russell diagram locus of a particularly young class of massive stars, the socalled Vz luminosity class, as they are arriving at the zero age main sequence.

The heavy dust content of the HEBs calls for doing high spatial resolution observations in the near infrared. Our aim in using the VLT/ISAAC was to detect and study all massive embedded stars inside N81, as well as the associated surrounding population. However, due to the limited spatial resolution of the present observations, this paper is mainly focused on the properties of the stellar environment of N81.

\section{Observations and data reduction}

The N81 region was observed in service mode with the ESO Very Large Telescope (VLT). The infrared spectro-imager ISAAC was used at the Nasmyth B focus of Antu through filters $K$ s on 7 October and $J$ and $H$ on 18 November 2001. The infrared detector (Hawaii Rockwell array) had $1024 \times$ 1024 pixels of $18.5 \mu \mathrm{m}$ each ( 0 .' 148 on the sky), thus providing a field of $2.5 \times 2$ '.5. The seeing varied between $1^{\prime \prime} .02$ and $1^{\prime \prime} .40$ $(F W H M)$.

A set of individual, 10-s exposures were obtained in each filter using a dithering method with a random offset of $15^{\prime \prime}$ at most. The number of exposures were 10, 20, and 18 for the $J$, $H$, and $K$ s bands respectively. The data were processed using the VLT pipeline and we verified that their quality was sufficient for our intended science. One area of concern was that small fluctuations of the sky near crowded areas of each frame could be due to sky subtraction by the pipeline "jitter" recipe. Therefore, we tried to supply the "jitter" with unbiased, flatfielded, sky-subtracted frames while disabling the sky calculation option of the recipe. Since this method reproduced the same fluctuation effects in the co-added frames, we decided to use the pipeline-reduced frames.

PSF-fitting photometry was carried out for all filters using the DAOPHOT II/ALLSTAR procedures under the ESO MIDAS reduction package. Finally the magnitudes were calibrated using the mean atmospheric extinction coefficients supplied by ESO, and three standard stars for determining the zero points. We used the color equations provided by ESO and checked that the color terms were small.

We compared our photometry with those provided by the 2MASS point source catalogue (Cutri et al. 2000) using a selection of 15 stars which appeared as single in our images and were brighter than $15.5 \mathrm{mag}$ in $K \mathrm{~s}$. Our photometry agrees well with 2MASS for $J$ and $H$ filters, although is slightly fainter; the mean differences being $m(2 \mathrm{MASS})-m($ ISAAC $)=$ $-0.05 \mathrm{mag}$ in $J$ and $-0.07 \mathrm{mag}$ in $H$. The disagreement is more significant for the $K \mathrm{~s}$ band, where the mean difference amounts to $-0.44 \mathrm{mag}$. The comparison of the filter profiles between ISAAC and 2MASS systems showed no significant difference. This discrepancy can therefore be explained by the fact that the $K$ s observations were carried out on a different night, as mentioned above. During that night the sky transparency was lower and the seeing poorer. We therefore decided to bootstrap our data with the 2MASS photometry by making the appropriate offset to our measured Ks magnitudes. Our final astrometry was based on 11 stars of the field, the accurate positions of which were determined in our previous HST observations (Paper I).

\section{Results and discussion}

A typical final image obtained with ISAAC is presented in Fig. 1, while a close-up view of the H II region is displayed in Fig. 2. The two brightest stars of N81 lying towards the central area of N81, detected by HST (Paper I) and separated by 0.27 , are not resolved on this image. The cross references for common detections in both ISAAC and HST are presented in Table 1. Even though we used broad band filters for our imaging in order to sample the properties of the stellar continuum emission, we do detect faint levels of diffuse near infrared light from the central $10^{\prime \prime}$ of the region. We note that the spatial extent of this emission is similar in size to that in $\mathrm{H} \alpha$ observed by HST (see Fig. 1 in Paper I). This is mainly due to nebular infrared emission lines (i.e. $\mathrm{Br} \gamma$ ) with contribution from free-free, two-photon, and probably dust emission. 


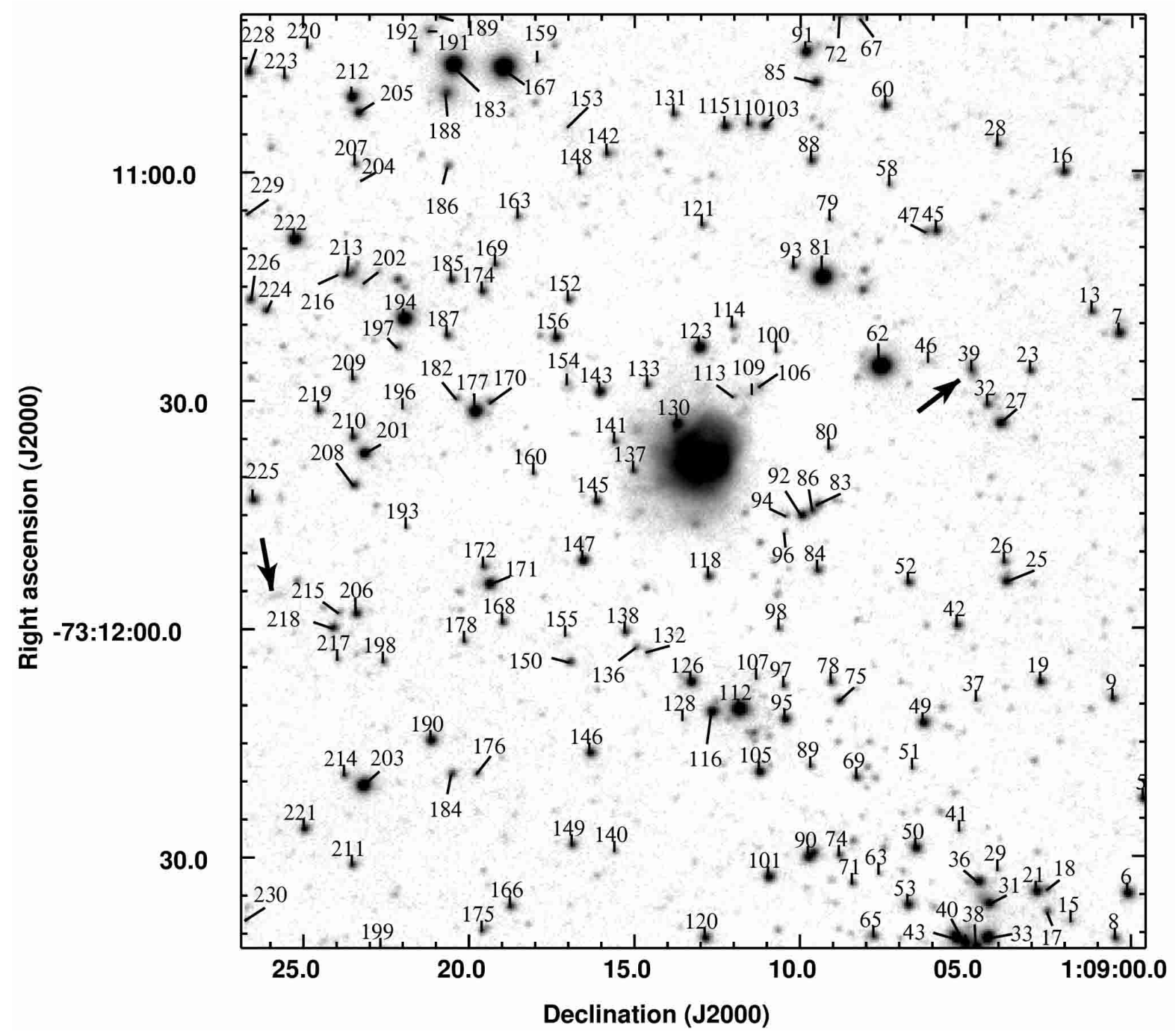

Fig. 1. The SMC N81 region and its surrounding field as seen through the $J$ filter. The image, taken with VLT/ISAAC, results from the coaddition of 10 basic exposures. The field size is $2^{\prime} \times 2^{\prime}$ corresponding to $38 \mathrm{pc} \times 38 \mathrm{pc}$. The arrows indicate the candidate background galaxies (Sect. 3.2). North is up and east to the left.

A total of 519 stars are detected in the field with magnitudes ranging from 12.5 to 20.7 in the $K$ s band. The internal photometric errors derived from DAOPHOT are very small ranging from 0.005 to $0.06 \mathrm{mag}$ for $K \mathrm{~s}$ from 14 to $19 \mathrm{mag}$ respectively. However, the true accuracy is smaller, a cutoff lower limit of $K \mathrm{~s}=19 \mathrm{mag}$ allowing an estimated accuracy of $0.2 \mathrm{mag}$ on the faintest stars.

\subsection{Color-magnitude and color-color diagrams}

In Fig. 3 we present the $K$ s versus $H-K$ s diagram of the observed stars in the field of N81. All sources brighter than 19th mag in $K \mathrm{~s}$ are present, and those brighter than $K \mathrm{~s}=$ 15 or redder than $H-K \mathrm{~s}=0.3 \mathrm{mag}$ are labelled according to Fig. 1. The color-magnitude diagram shows the presence of a main stellar population vertically distributed along $H-K \mathrm{~s} \sim$ $0.1 \mathrm{mag}$ and a second group with significantly redder colors.
There may also exist a third smaller population centered at $H-$ $K \mathrm{~s} \sim 0.0$, but we are not sure because of the photometric uncertainties. The populations are also visible on the color-color diagram (Fig. 4).

In order to explain the color-magnitude and color-color diagrams, we compared them with the predictions of the Geneva evolutionary models (Lejeune \& Schaerer 2001). To our surprise, we could not find any massive stars isochrones fitting both diagrams coherently. The best results were found for masses around 2 and $5 M_{\odot}$. Our analysis suggests that the bulk of the stars, distributed along $H-K \mathrm{~s} \sim 0.1 \mathrm{mag}$, belongs to an evolved population of mass $\sim 2 M_{\odot}$ and age 1 Gyr. Moreover, the $H-K \mathrm{~s} \sim 0.0$ population is simulated by $\sim 5 M_{\odot}$ model stars of age 1 Gyr. However, the discrimination between these two populations is difficult as it is very sensitive also to the interstellar extinction. A small differential extinction is sufficient to move stars from one population to the next. 

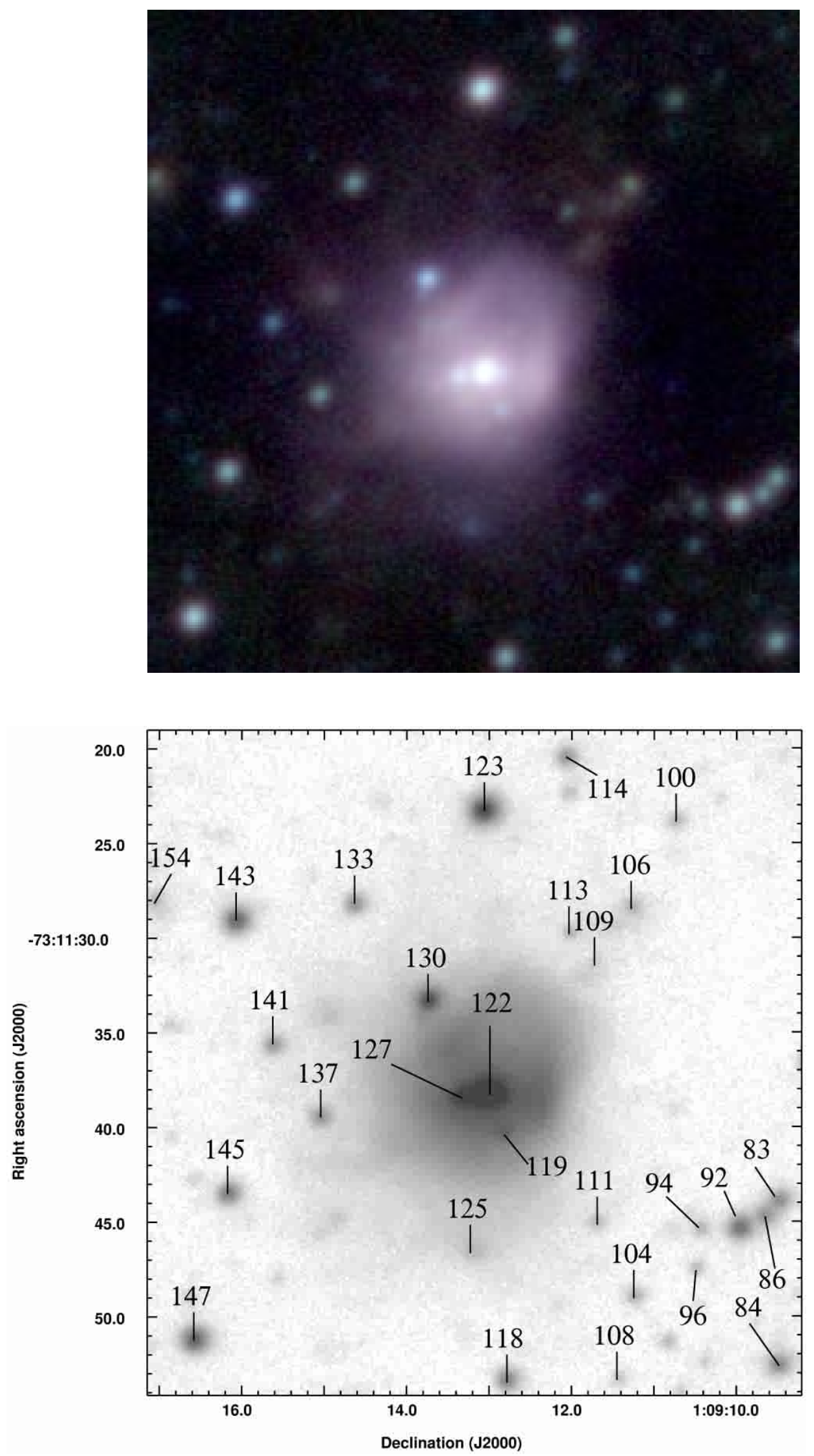

Fig. 2. The SMC H II region N81 and its immediate field. a) Composite $J H K$ s color image, b) as in a) with stars identified. 
Table 1. Photometry of common HST/ISAAC stars.

\begin{tabular}{|c|c|c|c|c|c|c|c|}
\hline Star & $\begin{array}{c}\alpha \\
(2000.0) \\
\end{array}$ & $\begin{array}{c}\delta \\
(2000.0) \\
\end{array}$ & $\begin{array}{c}J \\
(\mathrm{mag}) \\
\end{array}$ & $\begin{array}{c}H \\
(\mathrm{mag}) \\
\end{array}$ & $\begin{array}{c}K \\
(\mathrm{mag}) \\
\end{array}$ & $\begin{array}{c}I \\
(\mathrm{mag}) \\
\end{array}$ & $\begin{array}{c}H S T \text { number } \\
\text { (Paper I) }\end{array}$ \\
\hline 122 & 1:09:13.05 & $-73: 11: 38.2$ & 14.37 & 14.33 & 14.50 & 14.51 & $1+2$ \\
\hline 127 & 1:09:13.34 & $-73: 11: 38.4$ & 15.94 & 15.63 & 15.39 & 16.10 & 3 \\
\hline 119 & $1: 09: 12.83$ & $-73: 11: 40.2$ & 17.16 & 16.96 & 16.84 & 17.57 & 8 \\
\hline 130 & 1:09:13.74 & $-73: 11: 33.3$ & 16.21 & 16.25 & 16.43 & 16.11 & 11 \\
\hline 143 & 1:09:16.07 & $-73: 11: 29.1$ & 16.75 & 16.76 & 16.76 & 16.67 & 13 \\
\hline 141 & 1:09:15.62 & $-73: 11: 35.6$ & 18.24 & 18.16 & 18.25 & 18.14 & 14 \\
\hline 147 & 1:09:16.58 & $-73: 11: 51.2$ & 16.63 & 16.17 & 16.03 & 17.21 & 15 \\
\hline 111 & 1:09:11.70 & $-73: 11: 45.0$ & 19.34 & 19.18 & 19.10 & 19.07 & 17 \\
\hline 125 & 1:09:13.20 & $-73: 11: 46.5$ & 19.48 & 19.43 & 19.33 & 19.13 & 18 \\
\hline 118 & 1:09:12.78 & $-73: 11: 53.4$ & 17.64 & 17.10 & 16.96 & 18.49 & 19 \\
\hline 94 & 1:09:10.43 & $-73: 11: 45.4$ & 19.17 & 18.62 & 18.39 & 17.91 & 20 \\
\hline 92 & 1:09:09.96 & $-73: 11: 45.3$ & 17.09 & 16.48 & 16.30 & 18.85 & 21 \\
\hline 86 & 1:09:09.66 & $-73: 11: 44.7$ & 18.02 & 17.57 & 17.62 & 18.73 & 22 \\
\hline 137 & 1:09:15.05 & $-73: 11: 39.5$ & 18.17 & 17.58 & 17.55 & 19.11 & 23 \\
\hline 145 & 1:09:16.17 & $-73: 11: 43.5$ & 17.43 & 16.93 & 16.79 & 18.10 & 24 \\
\hline 133 & 1:09:14.63 & $-73: 11: 28.2$ & 18.05 & 17.50 & 17.42 & 18.68 & 26 \\
\hline 123 & 1:09:13.07 & $-73: 11: 23.3$ & 15.96 & 15.44 & 15.29 & 16.82 & 27 \\
\hline 95 & 1:09:10.48 & $-73: 12: 12.1$ & 17.03 & 17.06 & 17.11 & 19.66 & 29 \\
\hline 134 & 1:09:14.67 & $-73: 11: 54.8$ & 18.92 & 18.87 & 19.64 & 19.04 & 30 \\
\hline 96 & 1:09:10.49 & $-73: 11: 47.4$ & 19.44 & 19.03 & 18.90 & 19.78 & 33 \\
\hline 80 & 1:09:09.15 & $-73: 11: 36.5$ & 18.72 & 18.25 & 18.04 & 20.16 & 35 \\
\hline 203 & 1:09:23.22 & $-73: 12: 20.7$ & 15.44 & 15.22 & 15.08 & 15.84 & - \\
\hline 222 & 1:09:25.29 & $-73: 11: 08.9$ & 15.83 & 15.77 & 15.61 & 15.73 & - \\
\hline 201 & 1:09:23.19 & $-73: 11: 37.1$ & 16.38 & 15.77 & 15.53 & 17.36 & - \\
\hline 190 & 1:09:21.17 & $-73: 12: 14.8$ & 16.68 & 16.27 & 16.12 & 17.39 & - \\
\hline 212 & 1:09:23.55 & $-73: 10: 50.3$ & 16.35 & 15.93 & 15.77 & 16.97 & - \\
\hline 194 & 1:09:21.98 & $-73: 11: 19.4$ & 15.21 & 15.24 & 15.22 & 15.03 & - \\
\hline 171 & 1:09:19.41 & $-73: 11: 54.3$ & 16.37 & 16.19 & 15.87 & 16.47 & - \\
\hline 177 & 1:09:19.84 & $-73: 11: 31.6$ & 15.51 & 14.75 & 14.46 & 16.66 & - \\
\hline 183 & $1: 09: 20.51$ & $-73: 10: 46.0$ & 14.29 & 13.85 & 13.68 & 14.96 & - \\
\hline 126 & 1:09:13.32 & $-73: 12: 07.2$ & 16.48 & 15.84 & 15.67 & 17.49 & - \\
\hline 116 & 1:09:12.68 & $-73: 12: 11.1$ & 16.47 & 16.48 & 16.48 & 16.35 & - \\
\hline 112 & 1:09:11.87 & $-73: 12: 10.8$ & 14.98 & 14.98 & 14.99 & 14.87 & - \\
\hline 90 & 1:09:09.76 & $-73: 12: 30.2$ & 16.66 & 16.64 & 16.35 & 16.40 & - \\
\hline 49 & 1:09:06.30 & $-73: 12: 12.6$ & 16.77 & 16.33 & 16.22 & 17.57 & - \\
\hline 43 & 1:09:05.31 & $-73: 12: 40.8$ & 15.91 & 15.91 & 15.91 & 15.97 & - \\
\hline 40 & 1:09:05.03 & $-73: 12: 41.5$ & 16.25 & 16.22 & 16.24 & 16.19 & - \\
\hline 37 & 1:09:04.69 & $-73: 12: 41.9$ & 19.34 & 18.88 & 18.69 & 16.75 & - \\
\hline 36 & 1:09:04.62 & $-73: 12: 33.5$ & 16.84 & 16.79 & 16.75 & 16.86 & - \\
\hline 33 & 1:09:04.36 & $-73: 12: 40.9$ & 15.81 & 15.15 & 15.04 & 16.90 & - \\
\hline 21 & 1:09:02.87 & $-73: 12: 34.8$ & 16.75 & 16.79 & 16.87 & 16.69 & - \\
\hline
\end{tabular}

However, several previous works suggest that the interstellar extinction towards SMC N81 is rather low. This was also confirmed by the first high resolution extinction map of N81, derived from our HST observations (Paper I). Those observations showed that the extinction varies across N81, but the higher values do not exceed $A_{V} \sim 1.3 \mathrm{mag}$, while the mean value is $A_{V} \sim 0.40 \mathrm{mag}$, if the interstellar reddening law is used. Although this extinction is derived from the $\mathrm{H} \alpha / \mathrm{H} \beta$ Balmer decrement which could be biased towards less reddened regions, it has also been confirmed by measurements in the near infrared. In fact Israel \& Koornneef (1988) found $0<$ $A_{V} \leq 0.6$ from $\mathrm{Br} \gamma / \mathrm{H} \beta$ and $\mathrm{Br} \alpha / \mathrm{H} \beta$ ratios. We note also that the "nebular" Brackett/Balmer suffers, albeit in a lesser degree, from the same scattering out/in the beam versus $a b-$ sorption phenomenon as the Balmer/Balmer. However, a small Brackett/Balmer ratio does not necessarily imply a high extinction! The low extinction towards N81 is also supported by the fact that the color index $H-K$ s of the stars in our field does 


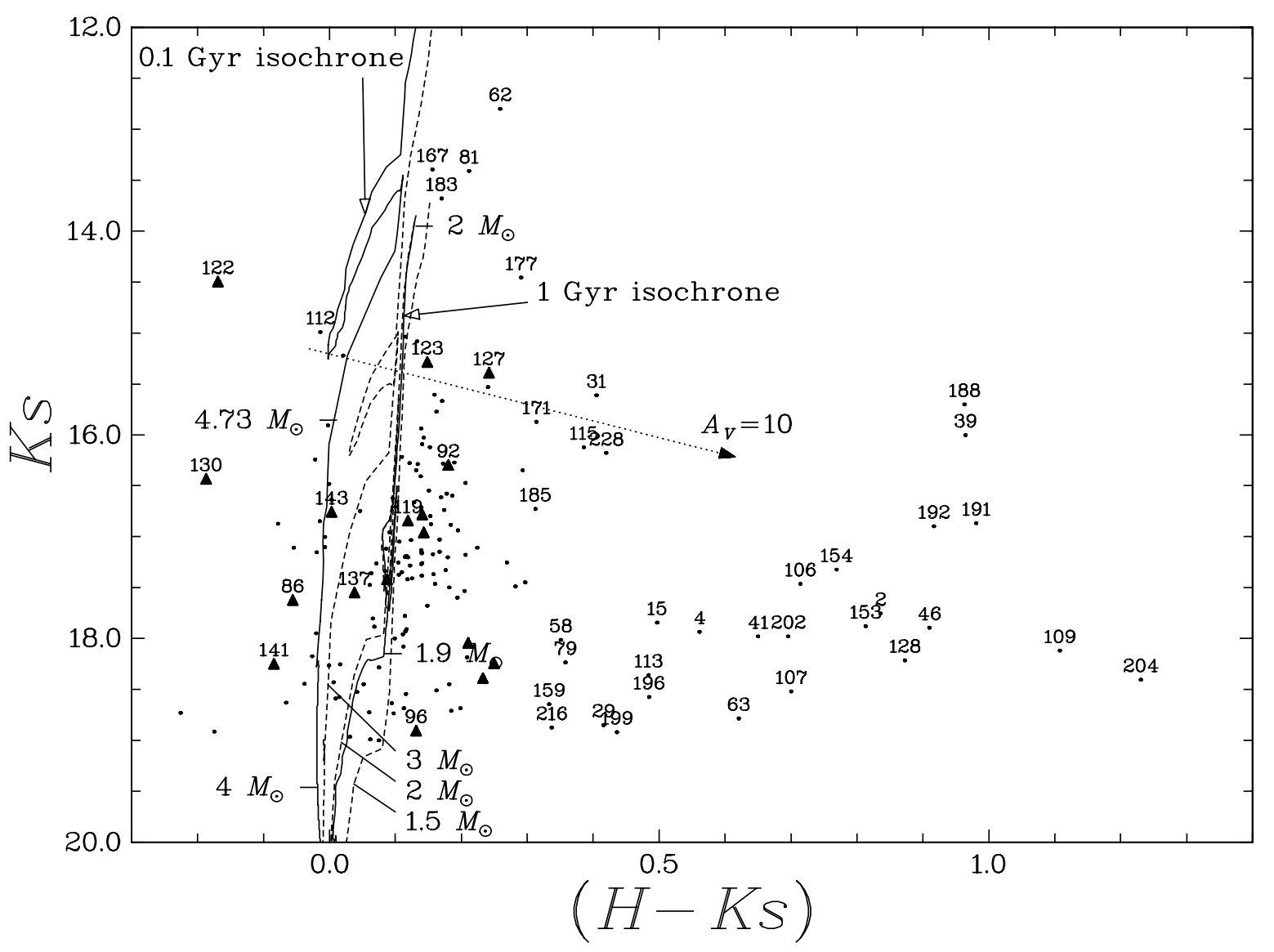

Fig. 3. Color-magnitude, $K$ s versus $H-K$ s, diagram for the observed stars towards SMC N81. The solid curves show isochrones predicted by the Geneva group (Lejeune \& Schaerer 2001). The left curve represents an age of $0.1 \mathrm{Gyr}$ and the right one 1 Gyr. Two mass boundaries are marked on each isochrone, 4 and $4.73 M_{\odot}$ on the left isochrone and 1.9 and $2 M_{\odot}$ on the right one. The dashed curves are evolutionary tracks for masses 1.5, 2, and $3 M_{\odot}$. The reddening track is plotted with a dotted line and extends to $A_{V}=10 \mathrm{mag}$. Note that an $A_{V}=10 \mathrm{mag}$, much too high for the area of SMC N81 (see text) would be needed for interstellar reddening to create the different color properties of the third stellar population we identified. The numbers refer to the stellar identifications presented in Fig. 1 and Table 1, and triangles represent stars that are also identified in Paper I.

not display any trend/correlation with the projected radial distance from N81 (Fig. 5). If N81 was responsible for the extreme reddening of some sources, one should expect them to lie in its vicinity. On the contrary, this plot shows that the reddest sources are randomly spread on the field, restricting the influence of N81 on the color of its neighbours to a radius of $5^{\prime \prime}$ at most. There is no evidence of segregation of red sources towards a particular area in our observed field.

Another argument against the presence of important dust concentration towards N81 comes from the fact that no important molecular cloud has been detected in the region. Israel et al. (1993) detected ${ }^{12} \mathrm{CO}(1-0)$ emission at two points towards N81 using SEST, even though the beamsize was $43^{\prime \prime}(\sim 13 \mathrm{pc})$, almost $\sim 4$ times the size of the H II region. The main $\mathrm{CO}$ emitting position, lying at the southwestern near periphery of the $\mathrm{H}$ II region, has a beam brightness temperature of $375 \mathrm{mK}$, a linewidth of $2.6 \mathrm{~km} \mathrm{~s}^{-1}$, an LSR velocity of $152 \mathrm{~km} \mathrm{~s}^{-1}$, and an intensity of $1.0 \mathrm{~K} \mathrm{~km} \mathrm{~s}^{-1}$. We tried to estimate the corresponding visual extinction from these molecular observations. Assuming molecular hydrogen densities of $1000-3000 \mathrm{~cm}^{-3}$, cloud temperatures of $10-40 \mathrm{~K}$, and a $\mathrm{CO} / \mathrm{H}_{2}$ abundance ratio of $10^{-5}$, we get $\mathrm{CO}$ column densities of $7-8 \times 10^{14} \mathrm{~cm}^{-2}$, corresponding to a visual extinction of $\sim 0.08 \mathrm{mag}$, if the beam is filled. A filling factor of $10 \%$ leads to column densities a factor of 10 larger and a visual extinction still smaller than 1 mag. Compared to other neighboring H II regions, the main $\mathrm{CO}$ position towards $\mathrm{N} 81$ is brighter than those detected towards N76, N78, and N80, but is weaker than that associated with N84 and more especially N88.

The disperse group of red stars we identified is not a result of extinction but they must represent a mix of evolved stars of the SMC lying along the line of sight of N81. The brightest star of the population, \#188, has a bolometric magnitude of $\sim 18.5 \mathrm{mag}$ which at the distance of the SMC translates to $400-500 L_{\odot}$. Consequently it very likely marks the tip of the red giant branch of the stellar population in the field of N81, even though we would need to obtain its spectrum to confirm this interpretation. Two other sources, \#109 and \#204, stand out with $H-K \mathrm{~s}>1$ and $K \mathrm{~s} \geq 18 \mathrm{mag}$ and luminosities of $\sim 50 L_{\odot}$. If this population actually consists of evolved red giants, it should be much older than the two other populations. Alternatively, these reddest colors may be due to circumstellar emission in young stellar objects, as found in the $\rho$ Oph molecular cloud (Greene \& Meyer 1995). However, since the 
M. Heydari-Malayeri et al.: SMC N81

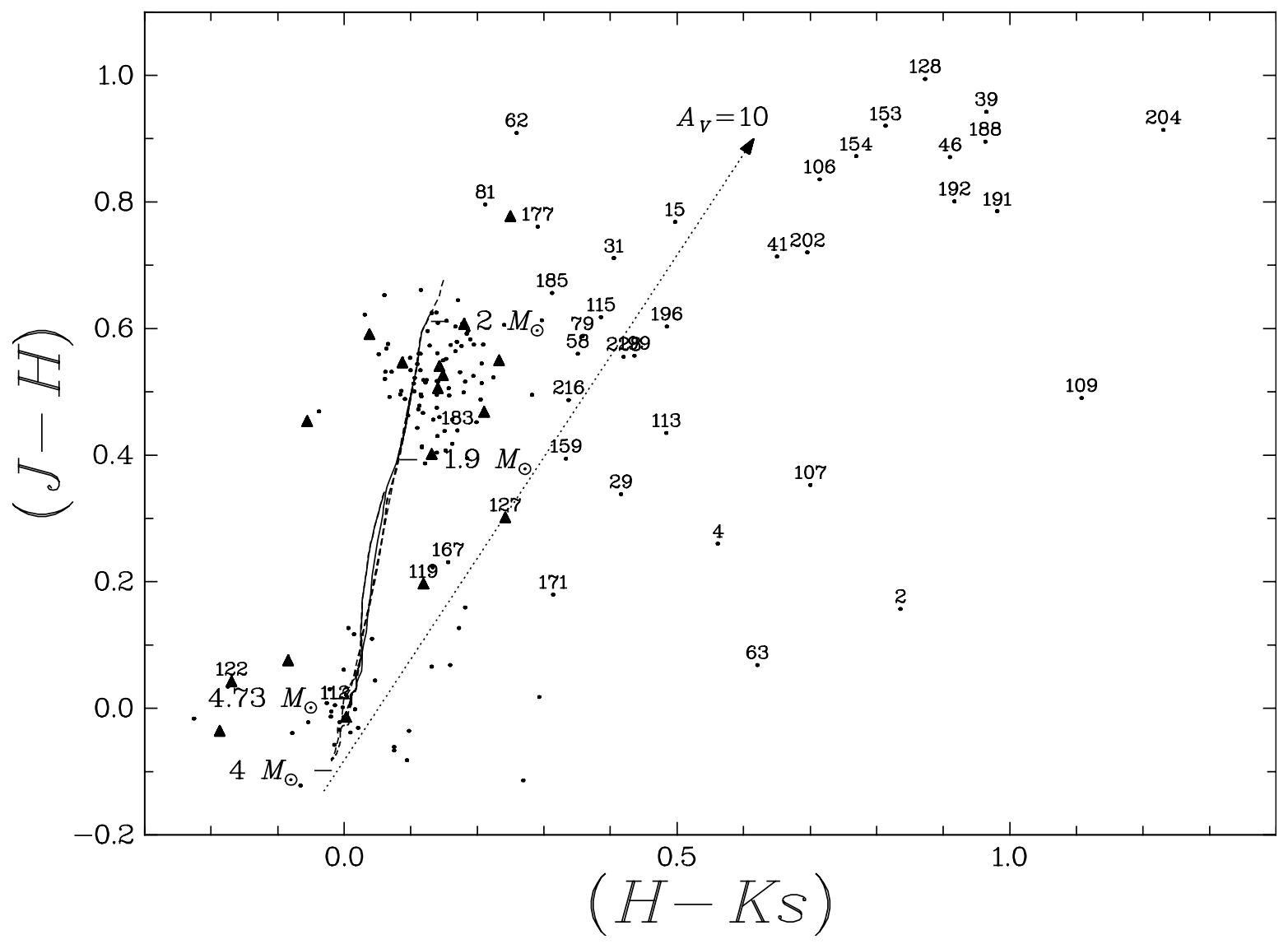

Fig. 4. Color-color, $J-H$ versus $H-K$ s, diagram for the observed SMC N81 stars. See the Fig. 3 caption for explanations regarding the model fits. Note that here the $0.1 \mathrm{Gyr}$ isochrone is indicated as a solid line and the $1 \mathrm{Gyr}$ curve as a dashed one. Triangles represent stars that are also identified in Paper I.

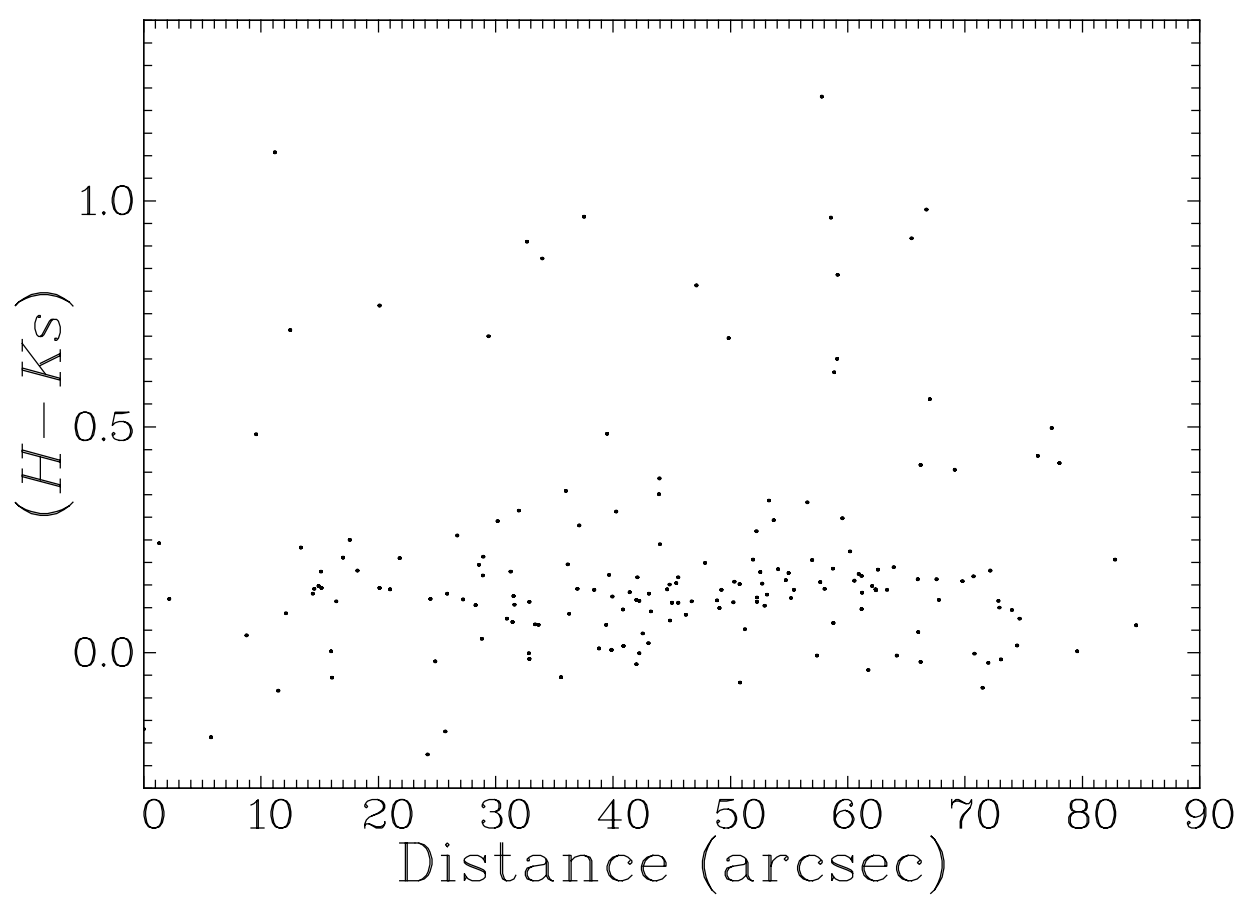

Fig. 5. Variation of color index $H-K \mathrm{~s}$ as a function of the angular distance from the center of N81. 
Table 2. Field stars comparison.

\begin{tabular}{rrrr}
\hline \hline Nebula & 2.5 & $5^{\prime}$ & $10^{\prime}$ \\
\hline SMC N81 & 56 & 359 & 1197 \\
N88 & 28 & 132 & 484 \\
N90 & 39 & 102 & 369 \\
N66 & 192 & 805 & 2883 \\
N70 & 142 & 581 & 2352 \\
\hline
\end{tabular}

extinction is very low towards SMC N81 and no important molecular clouds have been detected, this explanation seems less plausible.

In order to determine if our sample is contaminated by the background/foreground sources, we compared a few SMC fields through the 2MASS catalog. Using Aladin, we searched for sources around 5 nebulae (N81, N88, N90, N66, N70) for various surrounding areas (annuli with radii 2.5, 5, and 10 arcmin around the core objects), and compared the number of detections in each case (Table 2). The smallest annulus corresponds well with the typical size of a globular cluster in the SMC (Hodge 1985) and is roughly twice our ISAAC field. The HII regions N81, N88, and N90 lie in Shapley's wing, while N66 and N70 belong to the denser main body of the SMC and are much larger. In fact the stellar environment of N81 should preferably be compared with that of N88, since both are very young HEBs produced by newborn massive stars. Although N90 lies towards Shapley's wing, it is more extended, less excited and does not belong to the HEB class; it is probably older than N81 and N88A. Table 2 shows that the N81 field is richer than those of N88 and even N90, while we expected similar detection numbers based on their comparable evolutionary stages. Since N88 is associated with a large molecular cloud (Rubio et al. 1996) and is affected by a significantly stronger extinction, we argue that the higher number of stars detected towards N81 is due to our ability to probe deeper into the SMC in that region. We are in fact sampling all the stars belonging to different star formation events in that direction.

Previous work has shown the presence of large complexes of blue stars in the inter-Cloud region. Grondin et al. (1992) found associations as young as $16 \mathrm{Myr}$ with masses in the $1.5-12 M_{\odot}$ range, while Irwin et al. (1990) found older blue stars of age $\sim 0.1$ Gyr. However, these studies concern interCloud areas with Right Ascension $\alpha>2 \mathrm{~h}$, that is towards the Bridge central parts and significantly east of the N81 region. Tidal models predict that the Bridge was pulled from the SMC during a close encounter between the two clouds 0.2 Gyr ago (Gardiner \& Noguchi 1996), and the age of many of the stellar concentrations (10-25 Myr) indicates that the Bridge is a star forming region. The star populations identified in the present study are older than those previously detected in the Bridge region. They may have formed as a result of an earlier tidal interactions 1.5 Gyr ago (Murai \& Fujimoto 1980). This is consistent with Kunkel et al. (1997)'s result who found a population of intermediate age carbon stars, few Gyrs in age, scattered throughout the Bridge region. Model simulations suggest that the carbon stars are a population of the SMC halo stars displaced into the inter-Cloud region by tidal forces (Gardiner \& Noguchi 1996).

Finally, the main sequence massive stars identified using HST observations (Papers I and II) are also present on the color-magnitude and color-color diagrams. Shown with filled triangles in Figs. 3 and 4, they are mostly the bluest stars of the sample, while some are affected by local extinction.

\subsection{Observing background galaxies through SMC}

We have detected two non-stellar objects on the near infrared images. The source labelled \#39 (Fig. 1) is a rather bright, very reddened object with $K \mathrm{~s} \sim 16$ and $H-K \mathrm{~s}>0.9 \mathrm{mag}$. It has an elongated shape with an apparent size of 3 .' $5 \times 11^{\prime \prime} 5$, and is situated at the J2000 coordinates $\alpha=01: 09: 04.85, \delta=$ $-73: 11: 26.3$. It is very likely that this source be a background galaxy, but no extragalactic source at that location has been catalogued. The possibility of the object being a circumstellar disk can be ruled out because of its size which would be $2.2 \mathrm{pc}$, about 10 times larger than the circumstellar disks found for example in the Orion nebula (Brandner et al. 2000). Another possibility may be a blending of three aligned red stars. There is at least one additional "background galaxy" visible diagonally opposite to \#39 from the field centre, at coordinates $\alpha=$ 01:09:25.8, $\delta=-73: 11: 55.9$. These detections further underline the "transparency" of the SMC in that direction. It would be interesting to obtain spectra of these galaxy candidates in order to elucidate their nature.

\section{Conclusions}

The young massive star forming region N81, lying in Shapley's wing where the SMC bridges to the LMC, is surrounded by three main populations of lower mass stars. These are evolved stars of ages at least 0.1 to $1.0 \mathrm{Gyr}$ and initial masses of no more than $\sim 2$ to $5 M_{\odot}$. It appears that the newborn massive stars exciting the H II region N81 are not associated with these populations which presumably represent various star formation events in the SMC along the line of sight towards N81. Of course we cannot firmly exclude the co-spatiality of these populations with N81. However, the relative "transparency" of the SMC towards N81, as supported by this work, favors our interpretation, since the SMC is known to have an overall complex structure with several overlapping neutral hydrogen layers (McGee \& Newton 1981). There may exist a few runaway massive stars contaminating the low mass population, but the absence of any H II region in the field, apart from N81, is in line with the lack of high mass stars. Anyhow, the number of Magellanic Cloud main sequence stars counts for a small fraction of the total number of stars in the Wing region (Irwin et al. 1990).

\section{References}

Bouchet, P., Lequeux, J., Maurice, E., Prévot, L., \& Prévot-Burnichon, M. L. 1985, A\&A, 149, 330

Brandner, W., Grebel, E. K., Chu, Y., et al. 2000, AJ, 119, 292 
Caldwell, J. A. R., \& Coulson, I. M. 1986, MNRAS, 218, 223

Churchwell, E. 1990, A\&ARv, 2, 79

Cutri, R. M., et al. 2000, Explanatory Supplement to the 2MASS Second Incremental Data Release (Pasadena: Caltech)

Gardiner, L. T., \& Noguchi, M. 1996, MNRAS, 278, 191

Green, T. P., \& Meyer, M. R. 1995, ApJ, 450, 233

Grondin, L., Demers, S., \& Kunkel, W. E. 1992, AJ, 103, 1234

Henize, K. G. 1956, ApJS, 2, 315

Heydari-Malayeri, M., \& Testor, G. 1982, A\&A, 111, L11

Heydari-Malayeri, M., \& Testor, G. 1983, A\&A, 118, 116

Heydari-Malayeri, M., \& Testor, G. 1985, A\&A, 144, 98

Heydari-Malayeri, M., \& Testor, G. 1986, A\&A, 162, 180

Heydari-Malayeri, M., Le Bertre, T., \& Magain, P. 1988, A\&A, 195, 230

Heydari-Malayeri, M., van Drom, E., \& Leisy, P. 1990, A\&A, 240, 481

Heydari-Malayeri, M., Charmandaris, V., Deharveng, L., Rosa, M. R., \& Zinnecker, H. 1999a, A\&A, 347, 841

Heydari-Malayeri, M., Rosa, M. R., Charmandaris, V., Deharveng, L., \& Zinnecker, H. 1999b, A\&A, 352, 665

Heydari-Malayeri, M., Rosa, M. R., Zinnecker, H., Deharveng, L., \& Charmandaris, V. 1999c, A\&A, 344, 848 (Paper I)

Heydari-Malayeri, M., Charmandaris, V., Deharveng, L., et al. 2001a, A\&A, 372, 527

Heydari-Malayeri, M., Charmandaris, V., Deharveng, L., et al. 2001b, A\&A, 372, 495

Heydari-Malayeri, M., Charmandaris, V., Deharveng, L., et al. 2002a, A\&A, 381, 941

Heydari-Malayeri, M., Rosa, M. R., Schaerer, D., Martins, F., \& Charmandaris, V. 2002b, A\&A, 381, 951 (Paper II)
Hindman, J. V., Kerr, F. J., \& McGee, R. X. 1963, Aust. J. Phys., 16, 570

Hodge, P. 1985, PASP, 97, 530

Irwin, M. J., Demers, S., \& Kunkel, W. E. 1990, AJ, 99, 191

Israel, F. P., Johansson, L. E. B., Lequeux, J., et al. 1993, A\&A, 276, 25

Israel, F. P., \& Koornneef, J. 1988, A\&A, 190, 21

Israel, F. P., \& Koornneef, J. 1991, A\&A, 248, 404

Kerr, F. J., Hindman, J. V., \& Robinson, B. J. 1954, Aust. J. Phys., 7, 297

Kunkel, W. E., Irwin, M. J., \& Demers, S. 1997, A\&AS, 122, 463

Lejeune, T., \& Schaerer, D. 2001, A\&A, 366, 538

Martins, F., Schaerer, D., \& Heydari-Malayeri, M. 2002, in A Massive star Odyssey, from Main Sequence to Supernovae, IAU Symps. 212, ed. K. van der Hucht et al., 564

Mathewson, D. S., \& Ford, V. L. 1984, in Structure and Evolution of the Magellanic Clouds, IAU Symp., 108, 125

Mathewson, D. S., Ford, V. L., \& Visvanathan, N. 1986, ApJ, 301, 664

McGee, R. X., \& Newton, L. M. 1981, Proc. Astron. Soc. Aust., 4, 189

Moore, B., \& Davis, M. 1994, MNRAS, 270, 209

Murai, T., \& Fujimoto, M. 1980, PASJ, 32, 581

Putman, M. E., Gibson, B. K., Staveley-Smith, L., et al. 1998, Nature, 394, 752

Rubio, M., Lequeux, J., Boulanger, F., et al. 1996, A\&AS, 118, 263

Shapley, H. 1940, Harward Bull., 914, 8

Tenorio-Tagle, G., Yorke, H. W., \& Bodenheimer, P. 1979, A\&A, 80, 110

Testor, G., \& Pakull, M. 1985, A\&A, 145, 170 